journal club

Welche Frühsymptome lassen eine spätere Parkinson-Erkrankung vermuten?

\title{
Den Parkinson vorhersagen
}

Fragestellung: Welche Frühsymptome lassen das spätere Auftreten eines idiopathischen Parkinson-Syndroms vermuten?

Hintergrund: Parkinson-Patienten sollten früh therapiert werden, was eine Frühdiagnose der Erkrankung voraussetzt. Da zuverlässige Biomarker fehlen, untersuchten die Autoren, ob es klinische Symptome gibt, die auf das spätere Auftreten des idiopathischen Parkinson-Syndroms deuten. Die meisten Kollegen gehen davon aus, dass typische Frühsymptome Hyposmie, Obstipation, REM-Schlaf-Verhaltensstörung und Depression sind.

Patienten und Methodik: In Großbritannien sind im „The Health Improvement Network“ (THIN) anonymisiert die longitudinalen Krankengeschichten von mehr als elf Millionen Individuen aus mehr als 500 allgemeinärztlichen Praxen registriert. Dies entspricht etwa $6 \%$ der Gesamtpopulation. In diesen Unterlagen studierten die Autoren den zeitlichen Verlauf der klinischen Symptome von insgesamt 8.166 späteren Parkinson-Patienten und 46.755 altersentsprechenden Kontrollen von Anfang 1996 bis Ende 2012. Unter den Symptomen, nach denen die Autoren fahndeten, waren Tremor, Rigor, posturale Instabilität, Nackenschmerzen, Steifigkeit, Schulterschmerzen, Obstipation, Hypotension, erektile Dysfunktion, Blasendysfunktion, Schwindel, Gedächtnisschwäche, spät auftretende Angst oder Depression, zunehmende Vergesslichkeit, Apathie, Fatigue, Schlaflosigkeit, Anosmie, Hypersalivation und gestörter REM-Schlaf. Nur die Symptome wurden weiter verfolgt, die in mehr als $1 \%$ der Fälle pro

Schrag A, Horsfall L, Walters K et al. Prediagnostic presentations of Parkinson's disease in primary care: a case-control study. Lancet Neurol 2014; 14: 57-64
1.000 Personenjahre vorlagen. Die Risikorate wurde für Patienten mit und ohne Parkinson zwei, fünf und zehn Jahre vor der Diagnose berechnet.
Ergebnisse: Bereits fünf Jahre vor der endgültigen Diagnose war bei den späteren Parkinson-Patienten häufiger ein Tremor zu finden. Zwei Jahre vor der Diagnosestellung traf dies für $41 \%$ der späteren Parkinson-Patienten zu. Außerdem zeigten spätere Parkinson-Patienten zwei Jahre und fünf Jahre vor Diagnose häufiger eine posturale Instabilität. Schulterschmerz und Steifigkeit sowie Rigor waren ebenfalls zwei Jahre vor der Diagnosestellung deutlich häufiger bei den späteren Parkinson-Patienten. Kein signifikanter Unterschied konnte dagegen für Steifigkeit und Nackenschmerzen zwischen Kontrollen und späteren Parkinson-Patienten festgestellt werden. Bezüglich der neuropsychiatrischen Symptome fand sich bereits fünf Jahre vor der späteren Parkinson-Diagnose eine höhere Depressionsinzidenz. Zwei Jahre vor der Parkinson-Diagnose zeigte sich eine Depression bei $10 \%$ der späteren Parkinson-Patienten, aber nur bei $4 \%$ der Kontrollen. Für alle anderen neuropsychiatrischen Symptome gab es lediglich für Angst fünf und zwei Jahre vor der Diagnose und für Gedächtnisprobleme zwei Jahre vor Diagnose einen signifikanten Unterschied zu Ungunsten der späteren Parkinson-Patienten. Die Analyse der autonomen Nervenstörungen zeigte insbesondere für die Obstipation zwei Jahre vor Diagnose der Parkinson-Erkrankung eine signifikante Differenz zwischen den späteren Parkinson-Patienten und den Kontrollen (32\% vs. 19\%). Bereits zehn Jahre vor Diagnosestellung war die Obstipation häufiger bei späteren Parkinson-Patienten zu finden. Auch Schwindel, erektile Dysfunktion und Blasenstörungen waren häufiger. Zudem litten spätere Parkinson-Patienten bereits fünf Jahre vor späterer Diagnose signifikant häufiger unter Schlaflosigkeit und Müdigkeit (Fatigue).

Schlussfolgerungen: Eine Reihe klinischer Symptome deutet bereits Jahre vor der Diagnose eines idiopthischen ParkinsonSyndroms auf die spätere Erkrankung hin.

\section{- Kommentar von Heinz Reichmann, Dresden}

\section{Nicht alle prämotorischen Symptome wurden erfasst}

Die große Leistung dieser Studie ist, dass bei einer unglaublich hohen Anzahl an späteren Parkinson-Patienten und altersentsprechenden Kontrollen frühe Symptome der ParkinsonErkrankung evaluiert und detektiert werden konnten. Herausragend sind dabei die Symptome Tremor und Obstipation, wohingegen andere typische prämotorische Symptome wie REM-Schlaf-Verhaltensstörung und Hyposmie die Marge von über $1 \%$ pro 1.000 Patientenjahre zu meinem Erstaunen nicht erreicht haben. Hier muss allerdings auch kritisch eingewandt werden, dass die Mehrzahl aller Patienten über 50 Jahre alt war, sodass die Situation junger Patienten hier nicht untersucht werden konnte. Es ist auch nicht auszuschließen, dass Symptome wie Hyposmie und REM-Schlaf-Verhaltensstörung von den Hausärzten unterdurchschnittlich häufig diagnosti- ziert wurden, weil sie zum Beispiel von den Patienten nicht angegeben wurden und insbesondere weil nicht spezifisch danach gefragt wurde.

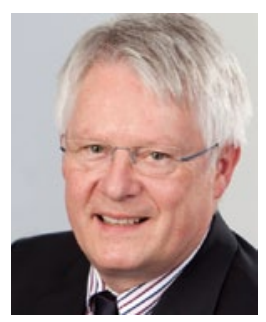

Prof. Dr. med. Heinz Reichmann, Dresden

Direktor der Klinik und Poliklinik für Neurologie, Klinikum Carl Gustav Carus der TU Dresden, Dekan der Medizinischen Fakultät. E-Mail: heinz.reichmann@ uniklinikum-dresden.de 